\title{
Determinación de dislipidemias en adultos mayores e intervención de enfermería sobre estilos de vida modificable en empleados civiles de la
}

FAP

Amalia Loli, Edna Ramírez, Miguel Sandoval, Aidé Casquero, Angélica Loli

Facultad de Medicina, UNMSM

Objetivos: Determinar la incidencia de dislipidemia e identificar el estilo de vida de los adultos mayores, empleados civiles de la Fuerza Aérea del Perú (FAP).

Diseño: Descriptivo, observacional.

Institución: Facultad de Medicina, UNMSM.

Participantes: Empleados civiles de la FAP.

Intervenciones: En la etapa clínica, 133 adultos fueron sometidos previo consentimiento a estudios clínicos: medidas antropométricas, presión arterial, análisis de sangre (colesterol total, HDL, LDL, triglicéridos y glucosa). En la etapa educativa se aplicó una encuesta y se realizó un programa de intervención de enfermería.

Principales medidas de los resultados: Medición de niveles antropométricos, estilos de vida mediante encuesta y niveles de presión arterial, colesterol HDL, LDL, triglicéridos y glucosa.

Resultados: El $45,1 \%(60)$ de los participantes tenía sobrepeso y 25,5\% (34) era obeso. El $45 \%$ (60) tomaba algún fármaco permanentemente. El $41,3 \%$ (55) realizaba caminatas diariamente. Sin embargo, el $63,2 \%(84)$ no realizaba deporte alguno. El $49,6 \%$ (66) manifestó comer verduras todos los días. El pollo y el pescado fueron las carnes que más consumían en la semana. Se detectó 74 casos de adultos con dislipidemia: 30 casos por elevación del colesterol, 22 casos por elevación de triglicéridos y 22 casos por elevación de ambos. El 28,5\% de los participantes tuvo el índice colesterol total /HDL mayor de 5, lo que significa riesgo cardiovascular.

Conclusiones: El 55\% de los adultos presentó dislipidemia. La mayoría de los adultos tenía sobrepeso y obesidad debido a poca actividad física. La mayoría no consumía frutas secas y no utilizaba aceite de oliva; sin embargo, ingería al menos una fruta al día y agua regularmente, así como verduras todos los días. Se recomienda intervención educativa individualizada para reforzar los hábitos y estilos de vida saludables de los adultos.

Palabras clave: Dislipidemia, adultos, intervención de enfermería. 